UCRL-ID-130769

\title{
Inverse Synthetic Aperture Radar Processing using Parametric Time-Frequency Estimators
}

\author{
Phase I Progress Report \\ (Sept. 97 -- Dec. 97)
}

\author{
J. V. Candy
}

B. Johnston

December 31, 1997

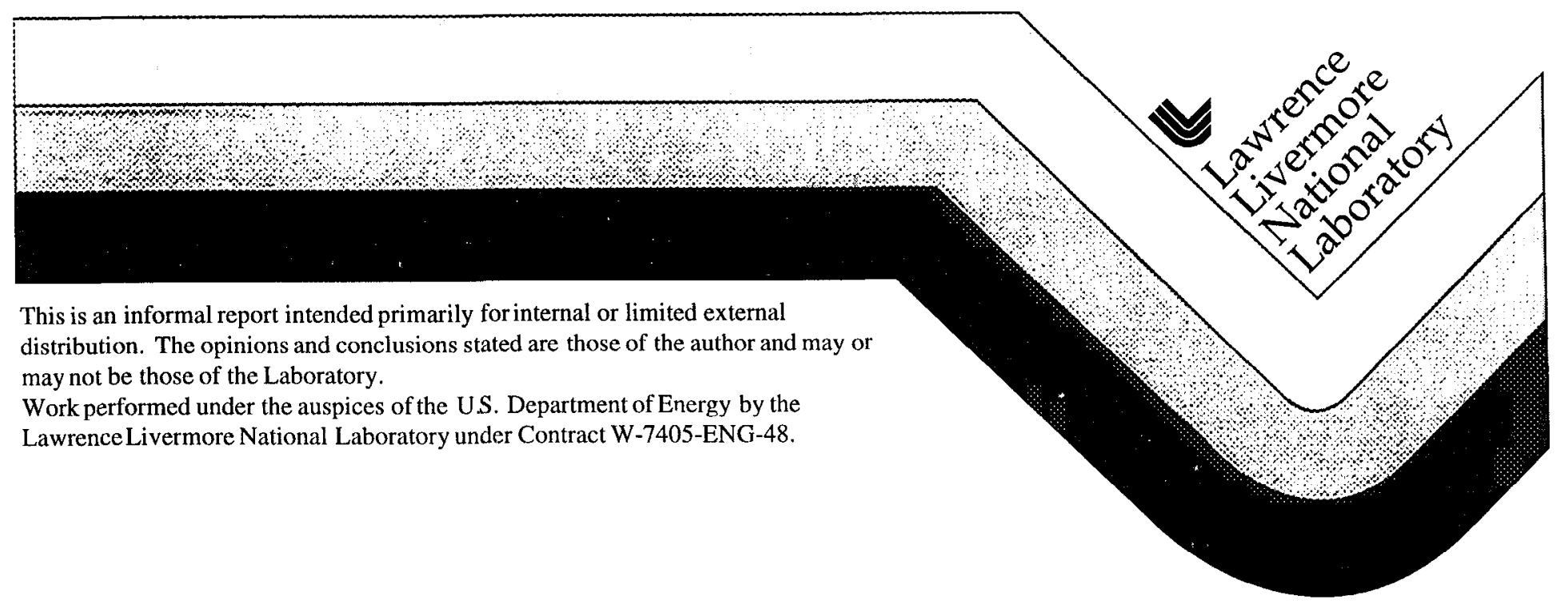




\section{DISCLAIMER}

This document was prepared as an account of work sponsored by an agency of the United States Government. Neither the United States Government nor the University of California nor any of their employees, makes any warranty, express or implied, or assumes any legal liability or responsibility for the accuracy, completeness, or usefulness of any information, apparatus, product, or process disclosed, or represents that its use would not infringe privately owned rights. Reference herein to any specific commercial product, process, or service by trade name, trademark, manufacturer, or otherwise, does not necessarily constitute or imply its endorsement, recommendation, or favoring by the United States Government or the University of California. The views and opinions of authors expressed herein do not necessarily state or reflect those of the United States Government or the University of California, and shall not be used for advertising or product endorsement purposes.

This report has been reproduced directly from the best avallable copy.

Available to DOE and DOE contractors from the

Office of Scientific and Technical Information

P.O. Box 62, Oak Ridge, TN 37831

Prices available from (423) 576-8401

Available to the public from the

National Technical Information Service

U.S. Department of Commerce

5285 Port Royal Rd.,

Springfield, VA 22161 
This report summarizes the work performed for the Office of the Chief of Naval Research (ONR) during the period of 1 September 1997 through 31 December 1997. The primary objective of this research was aimed at developing an alternative time-frequency approach which is recursive-intime to be applied to the Inverse Synthethic Aperture Radar (ISAR) imaging problem discussed subsequently. Our short term (Phase I) goals were to:

1. Develop an ISAR stepped-frequency waveform (SFWF) radar simulator based on a point scatterer vehicular target model incorporating both translational and rotational motion;

2. Develop a parametric, recursive-in-time approach to the ISAR target imaging problem;

3. Apply the standard time-frequency short-term Fourier transform (STFT) estimator, initially to a synthesized data set; and

4. Initiate the development of the recursive algorithm.

We have achieved all of these goals during the Phase I of the project and plan to complete the overall development, application and comparison of the parametric approach to other time-frequency estimators (STFT, etc.) on our synthesized vehicular data sets during the next phase of funding.

It should also be noted that we developed a batch minimum variance translational motion compensation (TMC) algorithm to estimate the radial components of target motion (see Section IV). This algorithm is easily extended to recursive solution and will probably become part of the overall recursive processing approach to solve the ISAR imaging problem.

Our goals for the continued effort are to:

1. Develop and extend a complex, recursive-in-time, timefrequency parameter estimator based on the recursive prediction error method (RPEM) using the underlying Gauss- Newton algorithms.

2. Apply the complex RPEM algorithm to synthesized ISAR data using the above simulator.

3. Compare the performance of the proposed algorithm to standard time-frequency estimators applied to the same data sets. 


\section{INTRODUCTION}

When a radar signal illuminates a target, the signal that is received is the superposition of the energy reflected or scattered by the target. The reflectivity function of this target determines its major features: size, shape, orientation, etc. in terms of providing a radar image mapped onto a range plane. The demands of high resolution imaging require large transmission bandwidths which are directly related to resolution in range. Typical broadband transmission signals employed by high resolution radar systems include frequency-modulated chirps as well as stepped-frequency waveforms. Resolution in cross-range (transverse to propagation) is determined by the antenna's beamwidth which is inversely proportional to its aperture. Larger apertures provide high cross-range resolution and therefore, more accurate radar images. Synthetic array radars include the synthetic aperture (SAR) in which the radar platform moves with fixed target position and its geometric inverse, the so-called inverse synthetic aperture (ISAR) in which the target is moving with the radar fixed [Cur91,Joh96,Rih96ab]. In this report we will concentrate our efforts on improved signal processing techniques for high resolution ISAR imaging developed from radar systems employing steppedfrequency transmitters.

The inverse synthetic aperture is formed by the coherent construction of signals obtained from the fixed aperture radar as the target translates and rotates "within its beamwidth", thus, creating the equivalent of a large circular aperture focused at the target's center-of-rotation (COR) [Che80,Wal80]. The underlying idea in ISAR imaging is to use the Doppler information provided by the different velocities relative to the radar of those parts of the target characterized as individual scatterers to obtain high crossrange resolution. That is, we decompose the target (spatially) into a set of individual "point" scatterers each of which has a different Doppler velocity represented by Doppler frequency shifts in the measured spectrum. Thus, the distribution of the target's reflectivity function can be measured by the Doppler spectrum. Typically, the Doppler spectrum is estimated using conventional Fourier transform techniques implemented computationally using the fast Fourier transform (FFT) algorithm with the underlying assumption that the Doppler frequency is fixed or time invariant. When the target moves this assumption is no longer valid and the Doppler spectrum is smeared degrading the cross-range resolution. In this work we investigate the application of recursive (in-time), parametric, time-frequency techniques [Lju87,Sod89,Coh95,Boa92] to improve the cross-range resolution of the radar and estimate the desired Doppler spectrum as opposed to the more traditional time-frequency, spectral estimators using the short time Fourier transform (STFT) or joint time-frequency (JTF) estimation techniques such as the Wigner-Ville or wavelet transforms [Coh95,Qia96]. We develop the background and underlying mathematics of the problem in Section II. In 
Section III we develop the basic time-frequency approach. Next we discuss the development of a simulation based on the point scatterer decomposition of the target function and apply the standard short-time Fourier transform (STFT) time-frequency estimator to the synthesized ISAR data. In Section V we develop the recursive-in-time algorithm, apply it to the synthesized data and compare the results with that of the STFT. We summarize our results and discuss future work in the final section.

\section{BACKGROUND}

In this section we briefly develop the underlying mathematics to define the ISAR problem based on the geometrical relations depicted in Figure 1 below. A cross-sectional reflectivity function for a typical vehicle is defined in terms of its center-of-rotation as shown. The geometry is defined in terms of the target's translational and rotational motion relative to the radar. That is, $\theta(t)$, is the instantaneous rotational angle in the XY-coordinate system with the radar line-of-sight (LOS) along the Y-axis defining its instantaneous slant range, $R(t)$. The instantaneous range of the $i$-th point scatterer at $\left(x_{i}, y_{i}\right)$ is defined by $r_{i}(t)$, while the target itself is defined by its reflectivity function, $\rho(x, y)$, shown in two dimensions (2D). Radar imaging can be thought of as the estimation of the 2D (or 3D) reflectivity function from a set of temporal radar measurements or returns, $\{\tilde{m}(t)\}$.

Suppose a radar emits a monotone or single frequency, $f$ (in $\mathrm{MHz}$ ), unit pulse, $p(t)$, of duration, $T_{p}$, given by

$$
p(t)=e^{j 2 \pi f t}, \quad 0 \leq t \leq T_{p} .
$$

After the target is illuminated by the field, it reflects or scatters the transmitted 'energy in a differential area, $(d x, d y)$, back to the radar receiver which is proportional to

$$
\text { received energy } \propto \rho(x, y) e^{j 2 \pi f(t-\tau)} d x d y, \quad \frac{2 R}{c} \leq t \leq T_{p}+\frac{2 R}{c},
$$

where $\rho(x, y)$ is the target reflectivity function, $c$ is the wave propagation speed and $\tau$ is the round-trip pulse travel time

$$
\tau=\frac{2 R}{c} .
$$

The received echo from the target measured by the radar system after normalization (gain, amplitude, attenuation, etc.) is therefore 


$$
\tilde{m}(t)=\int_{-\infty}^{\infty} \int_{-\infty}^{\infty} \rho(x, y) e^{j 2 \pi f(t-\tau)} d x d y, \quad \frac{2 R}{c} \leq t \leq T_{p}+\frac{2 R}{c}
$$

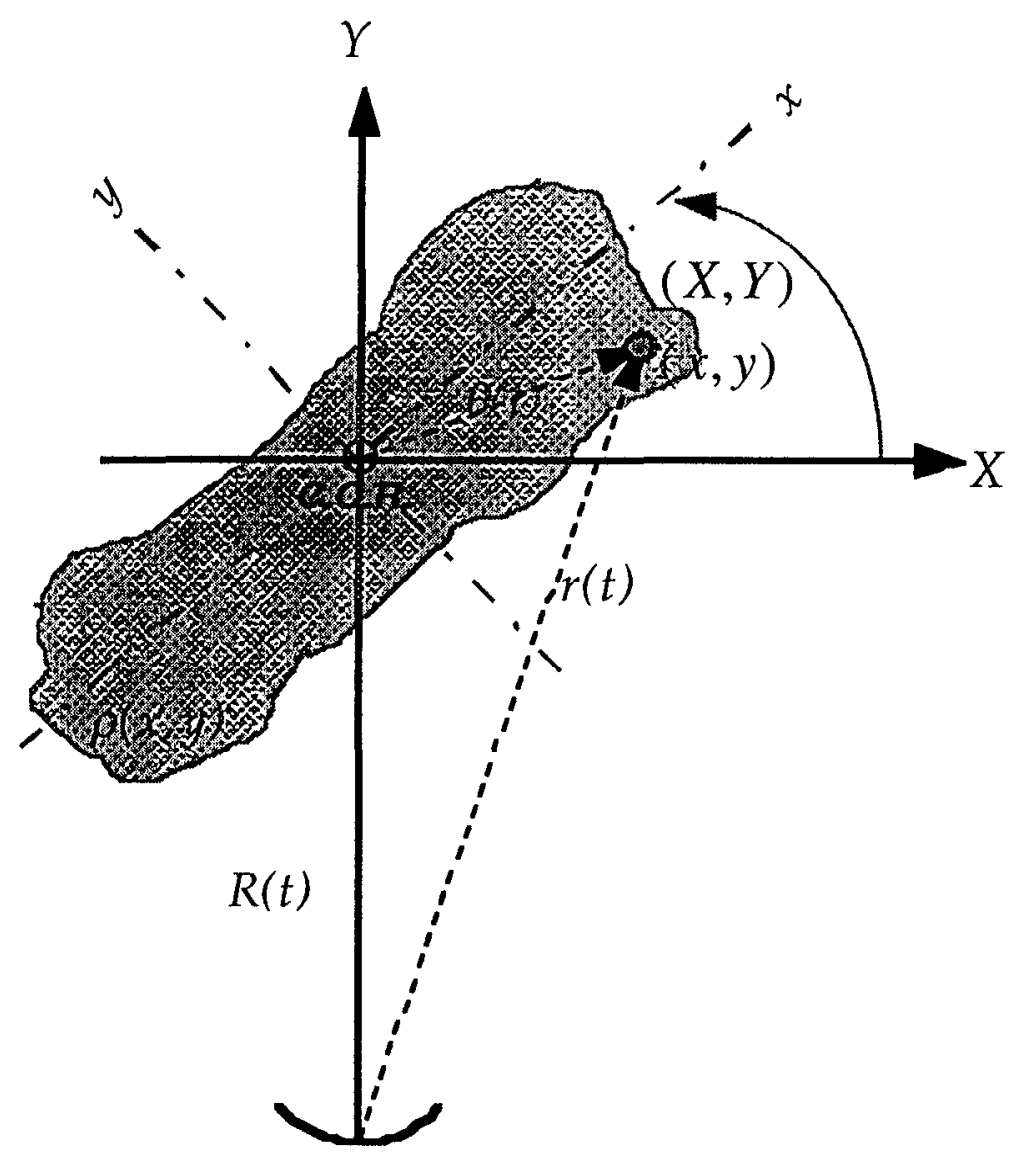

Figure 1. ISAR Geometry: Target and Scatterer at location $(x, y)$.

Baseband processing removes the monotone, $e^{j 2 \pi f t}$, through mixing to give the superposition of all the point scatterers composing the target reflectivity yielding the radar measurement,

$$
m(t)=\int_{-\infty}^{\infty} \int_{-\infty}^{\infty} \rho(x, y) e^{-j 2 \pi f \tau} d x d y
$$

Now return to the ISAR geometry of Figure 1 and allow the target to have both translational and rotational motion in terms of the $X Y$-coordinate 
system, $R(t)$, and $\theta(t)$, respectively. We can represent these functions in terms of their Taylor series expansions as:

$$
R(t)=R_{o}+v_{R} t+\frac{1}{2} a_{R} t^{2}+H . O . T .
$$

with $R_{o}, v_{R}, a_{R}$ the respective initial range, target radial velocity and acceleration. Similarly, the rotation angle is given by:

$$
\theta(t)=\theta_{o}+\omega t+\frac{1}{2} \alpha t^{2}+H . O . T .
$$

and $\theta_{o}, \omega, \alpha$ are the respective initial angle, angular velocity and angular acceleration.

If we assume that the target dimensions are small relative to its range, $R(t)$, from the radar, that is, $R \gg>\max d_{i}$, for the $i$-th scatterer location, $d_{i}=\sqrt{x_{i}^{2}+y_{i}^{2}}$, then the instantaneous range of any scatterer at $(x, y)$, in terms of the local $x y$-coordinate system is

$$
r(t) \approx R(t)+y(t)=R(t)+(x \cos \theta(t)-y \sin \theta(t)) .
$$

Substituting this expression into Eq. 4 gives

$$
m(t)=\int_{-\infty}^{\infty} \int_{-\infty}^{\infty} \rho(x, y) e^{-j \frac{2 \pi f}{c}(R(t)+x \cos \theta(t)-y \sin \theta(t))} d x d y,
$$

or simplifying we obtain the radar measurement

$$
\begin{aligned}
m(t)=e^{-j \frac{2 \pi f R(t)}{c}} \int_{-\infty}^{\infty} \int_{-\infty}^{\infty} \rho(x, y) e^{-j 2 \pi\left(x \kappa_{x}(t)-y \kappa_{y}(t)\right)} d x d y, \\
\kappa_{x}(t) \equiv \frac{2 f}{c} \cos \theta(t), \\
\kappa_{y}(t) \equiv \frac{2 f}{c} \sin \theta(t) .
\end{aligned}
$$

where $\kappa_{x}, \kappa_{y}$ are the respective (spatial) wavenumbers.

Examining Eq. 8 more closely, we see that the first term is the phase due to the target translation in range while the second term is the twodimensional spatial Fourier transform (2DFT) of the target reflectivity function parameterized by $t$ and defined by 


$$
\mathrm{P}(\vec{\kappa} ; t) \equiv \int_{-\infty-\infty}^{\infty} \int_{-\infty}^{\infty} \rho(\vec{x}) e^{-j 2 \pi \vec{\kappa} \cdot \vec{x}} d \vec{x}
$$

where $\vec{\kappa} \cdot \vec{x}$ is the usual Euclidean inner product with $\vec{\kappa}=\left[\begin{array}{l}\kappa_{x} \\ \kappa_{y}\end{array}\right]$ and $\vec{x}=\left[\begin{array}{l}x \\ y\end{array}\right]$. Therefore we can express the radar measurement simply as

$$
m(t)=e^{-j \frac{2 \pi f R(t)}{c}} \mathrm{P}(\vec{\kappa} ; t),
$$

which shows that the target image or equivalently reflectivity function could be recovered by removing the target translational motion and taking the inverse 2DFT. We will discuss the details of the processing in Section III of this report.

The Doppler frequency shift induced by the target's motion can be obtained by differentiating the phase of the radar measurement with respect to time. The phase function of Eq. 8 is given by

$$
\varphi(f, t)=\frac{4 \pi f}{c} R(t)+x \kappa_{x}(t)-y \kappa_{y}(t)
$$

is therefore

$$
\frac{d \varphi(f, t)}{d t}=\frac{4 \pi f}{c} \dot{R}(t)+x \dot{\kappa}_{x}(t)-y \dot{\kappa}_{y}(t)
$$

From Eq. 6 we have

$$
\dot{R}(t)=v_{R}+a_{R} t
$$

and the Doppler frequency shift due to translational motion is therefore

$$
f_{D_{\text {trans }}}=\frac{1}{2 \pi}\left(\frac{4 \pi f}{c}\left(v_{R}+a_{R} t\right)\right)=\frac{2 f}{c}\left(v_{R}+a_{R} t\right)
$$

which shows that the translational motion induces a time-varying Doppler shift, if the velocity is not constant. Correspondingly, the Doppler induced by the rotational motion is given in terms of the spatial wavenumbers, since 


$$
\begin{aligned}
& \dot{\kappa}_{x}(t)=-\frac{2 f}{c} \dot{\theta}(t) \sin \theta(t)=-\frac{2 f}{c}(\omega+\alpha t) \sin \theta(t), \\
& \dot{\kappa}_{y}(t)=\frac{2 f}{c} \dot{\theta}(t) \cos \theta(t)=\frac{2 f}{c}(\omega+\alpha t) \cos \theta(t),
\end{aligned}
$$

and therefore the time-varying rotationa' Doppler shift is given by

$$
f_{D_{\text {rot }}}=-\frac{2 f}{c}(\omega+\alpha t)(x \sin \theta(t)+y \cos \theta(t)) \text {. }
$$

Thus, we see that the Doppler of each individual scatterer is induced by its translational and rotational motion. Since this shift is related to the geometric position of the individual scatterer at $(x, y)$ another scatterer at a different location within the target will have a different Doppler enabling it to be distinguished from the others.

Next we will decompose the radar measurement when we use a stepped-frequency rather than monotone pulse transmitter. The steppedfrequency radar transmits a sequence of $N$-bursts of $M$-narrowband (frequency) pulses in which the center frequency of each successive pulse is increased by a constant frequency step, $\Delta f$,

$$
f_{m}=f_{c}+m \Delta f, \quad m=0, \cdots, M-1
$$

at the carrier frequency, $f_{c}$. The total bandwidth of a burst specifies the slant range resolution, $\Delta R_{s r}$, by

$$
\Delta R_{s r}=\frac{c}{2 B W}=\frac{c}{2(M \Delta f)}
$$

and the total number of bursts, $N$, specifies the cross-range resolution as

$$
\Delta R_{c r}=\frac{\lambda}{2 \omega \mathrm{T}}
$$

where $\lambda$ is the radar wavelength $\left(\frac{1}{f_{c}}\right), \omega$ is the angular velocity of the target, and $\mathrm{T}$, is the observation time which is specified by the radar pulse repetition rate. Thus, for a stepped-frequency radar, the transmitted burst of 
narrowband pulses or equivalently the Stepped Frequency Waveform (SFWF) is given by

$$
p_{m}\left(t_{n}\right)=e^{j 2 \pi f_{m} t_{n}}, \quad m=0, \cdots, M-1 ; \quad n=0, \cdots, N-1,
$$

and therefore, the received signal of Eq. 10 is a temporal frequency domain signal given by

$$
M\left(f_{m}\right)=e^{\frac{-j 2 \pi f_{m} R\left(t_{n}\right)}{c}} \mathrm{P}\left(\vec{\kappa} ; t_{n}\right), \quad m=0, \cdots, M-1 ; \quad n=0, \cdots, N-1,
$$

which is a set of M-Fourier coefficients $\left\{M\left(f_{m}\right)\right\}, m=0, \cdots, M-1$; at time $t_{n}$. Suppose we multiply both sides of Eq. 21 by $e^{j 2 \pi f_{m} t_{n}}$ and sum over $m$, then we have

$$
\sum_{m=0}^{M-1} M\left(f_{m}\right) e^{j 2 \pi f_{m} t_{n}}=\sum_{m=0}^{M-1} e^{\frac{-j 2 \pi f_{m}\left(R\left(t_{n}\right)-t_{n}\right)}{c}} \mathrm{P}\left(\vec{\kappa} ; t_{n}\right)
$$

which is simply the inverse discrete Fourier transform (IDFT) of $M\left(f_{m}\right)$, or more precisely,

$$
m\left(t_{n}\right) \equiv \operatorname{IDFT}\left[M\left(f_{m}\right)\right]=\operatorname{IDFT}\left[e^{\frac{-j 2 \pi f_{m} R\left(t_{n}\right)}{c}} \mathrm{P}\left(\vec{\kappa} ; t_{n}\right)\right], \quad n=0, \cdots, N-1 .
$$

Thus, we see that the stepped-frequency radar measurement actually returns the target reflectivity response samples in the temporal frequency domain. Performing the IDFT on the measured data transforms it to the equivalent complex range profile space (range Vs. time). However, the usual processing is to first compensate for the range translational term (if known) and then perform the IDFT which would lead us back to Eq. 10 with the translational term removed. We discuss this in Section III.

Finally, let us return to the expected Doppler returns from a steppedfrequency radar. For a single burst pulse at $t_{n}$, we simply replace $t \rightarrow t_{n}$ in Eqs. 5 and 6; however, for a set of N-bursts we have $t \rightarrow t_{m, n}$ corresponding to the $\mathrm{m}$-th narrowband frequency pulse of the $\mathrm{n}$-th burst (see Weh95 for more details). This completes the required background, next we discuss the ISAR simulation. 


\section{ISAR SIGNAL PROCESSING}

In this section we discuss the basic ISAR signal processing which is employed to enhance the resolution of the point scatterers representing our moving vehicular target. The usual approach of conventional (chirped) processors is to perform range compression through matched-filtering [Cur91,Joh96] and then use the Fourier transform to retrieve the Doppler or equivalently cross-range information. The underlying assumption for successful application of this approach is that all scattterers remain in their range cells and that the corresponding Doppler frequency shifts are constant during imaging. As observed from Eqs. 14 and 16, the Doppler shifts for a moving target vary as a function of time; therefore, the Doppler spectrum will be smeared blurring the radar image. The development of an instantaneous time-frequency estimator alleviates this problem [Che97], and the typical 2D range/Doppler image becomes a 3D time-range-Doppler cube. Note that instead of applying a sophisticated rotational compensation estimator to correct for the motion, this time-frequency approach improves classical performance at each time step, enabling the observation of high resolution target images at each frame.

The basic signal processing operations performed on the measured ISAR (complex) data set obtained from a stepped-frequency radar are depicted in Figure 2. Due to the swept frequency transmission, the frequency domain data set obtained is defined in terms of the $m$-th frequency of the $n$-th burst as $\left\{M_{n}\left(f_{m}\right)\right\}, m=0, \cdots, M-1 ; n=0, \cdots, N-1$ or if we use vector notation, we have the set of burst vectors defined by

$$
\underline{M}_{n}(f) \equiv\left[\begin{array}{c}
M_{n}\left(f_{o}\right) \\
\vdots \\
M_{n}\left(f_{M-1}\right)
\end{array}\right] ; \quad n=0, \cdots, N-1
$$

where $\underline{M}_{n} \in \mathrm{C}^{M \times 1}$. After baseband processing, the range term in Eq. 21 must be compensated for by conjugate multiplication at each frequency step leading to translational motion compensation (TMC) which compensates for the gross effects of range by correcting for the translational motion of the target COR, that is,

$$
\underline{P}\left(\vec{\kappa}, t_{n}\right) \equiv\left[\begin{array}{c}
e^{+j 2 \pi f_{0} R\left(t_{n}\right)} \\
\vdots \\
e^{+j 2 \pi f_{M} R\left(t_{n}\right)}
\end{array}\right] \otimes \underline{M}_{n}(f) ; \quad n=0, \cdots, N-1 ;
$$


with $\otimes$ the Kronecker multiplication (element-by-element) and $\underline{P} \in \mathbf{C}^{M \times 1}$, the target reflectivity wavenumber-time spectrum. Once the TMC is accomplished, the target reflectivity function can be extracted from its spectrum through a 2D Fourier transform operation performed on $\mathrm{P}(\vec{\kappa}, f) \stackrel{F_{I I}^{-1}}{\rightarrow} \rho(\vec{x}, t)$. Decomposing this operation into individual steps or $1 \mathrm{D}$ operations on the columns and rows leads to the desired target reflectivity function or equivalently range/cross-range, or range/Doppler image. Following Figure 2, we apply the inverse discrete Fourier transform (IDFT) on the basebanded, TMC measurement data of Eq. 24 to yield the desired range profiles as

$$
\underline{\rho}\left(R_{s r}, t_{n}\right) \equiv \operatorname{IDFT}\left[\underline{\mathrm{P}}\left(\vec{\kappa}, t_{n}\right)\right], \quad n=0, \cdots, N-1,
$$

where the slant range is defined in the usual manner as $R_{s r}=\sqrt{x^{2}+y^{2}}$ which is a direct result of the columnwise transform.

We note that the TMC in the above formulation is performed in the measurement or temporal frequency domain provided by the SFWF radar; however, the underlying assumption inherent in this approach is that the time dependent range, $R\left(t_{n}\right)$, is known a-priori enabling the correction to be performed (see [Weh95] for details). An alternative is to first perform the IDFT on the raw SFWF radar data (as in Eq. 22) essentially providing uncompensated range profiles (see Figure 5), that is,

$$
\underline{\tilde{M}}\left(R_{s r}, t_{n}\right) \equiv I D F T\left[\underline{M}_{n}(f)\right] ; \quad n=0, \cdots N-1 .
$$

Next the time dependent range, $\hat{R}\left(t_{n}\right)$, is estimated from this uncompensated profile data using a minimum variance estimator discussed subsequently in Section IV and the equivalent TMC is performed to yield identical range profiles as

$$
\underline{\rho}\left(R_{s r}, t_{n}\right)=\left[\begin{array}{c}
e^{+j 2 \pi f_{0} R\left(t_{n}\right)} \\
\vdots \\
e^{+j 2 \pi f_{M-1} R\left(t_{n}\right)}
\end{array}\right] \otimes \underline{\tilde{M}}\left(R_{s r}, t_{n}\right) ; \quad n=0, \cdots, N-1 ;
$$

Equivalent frequency domain TMC algorithms have been developed [Weh95], but in this effort we choose to operate in the range profile (temporal) domain enabling the applicability of our approach to chirp or other type radar systems which collect range profile data directly. 
Using either TMC approach, the M-dimensional wavenumber-time reflectivity vectors are transformed into the range-time domain. To be more specific, we define the temporal reflectivity matrix as

$$
\mathrm{P}\left(R_{s r}, t\right) \equiv\left[\begin{array}{c}
\underline{\rho}^{T}\left(R_{o}, t\right) \\
\vdots \\
\underline{\rho}^{T}\left(R_{M-1}, t\right)
\end{array}\right]=\left[\begin{array}{lll}
\underline{\rho}\left(R_{s r}, t_{o}\right) & \cdots & \underline{\rho}\left(R_{s r}, t_{N-1}\right)
\end{array}\right] .
$$

The $\mathrm{N}$-dimensional rows of this matrix are the $m$-th reflectivity range cell time series, $\underline{\rho}^{T}\left(R_{m}, t\right) \in \mathbf{C}^{1 \times N}$ or equivalently the M-dimensional columns are the $n$-th reflectivity range profile given by the IDFT operation as $\rho\left(R_{s r}, t_{n}\right) \in \mathbf{C}^{M \times 1}$. Thus, the synthetic range profile data is defined in terms of the target reflectivity. Next Doppler processing is performed.

First, we assume that the target is slowly moving and the wavenumbers are approximately constant so that

$$
\begin{aligned}
& \kappa_{x}(t)=\frac{2 \pi f_{m}}{c} \cos \theta(t) \rightarrow \frac{2 \pi f_{m}}{c} \cos \theta \\
& \kappa_{y}(t)=\frac{2 \pi f_{m}}{c} \sin \theta(t) \rightarrow \frac{2 \pi f_{m}}{c} \sin \theta
\end{aligned}
$$

therefore, the temporal discrete Fourier transform (DFT) of the range profiles lead to the following range/Doppler or equivalently range/cross-range image,

$$
\rho\left(R_{s r}, R_{c r}\right)=\mathrm{P}\left(R_{s r}, f_{D}\right) \equiv D F T\left[\mathrm{P}\left(R_{s r}, t\right)\right]
$$

since the Doppler frequency is directly related to the cross-range. In fact, the direct relation between Doppler and cross-range enables the spectral analysis of the range cell time series to separate the contribution of individual scatterers---assuming constant Doppler.

As before, the frequency resolution is inversely proportional to the processing time duration $T$, therefore, Eq. 19 implies that

$$
f_{D}=\frac{2 \omega}{\lambda} R_{c r}
$$




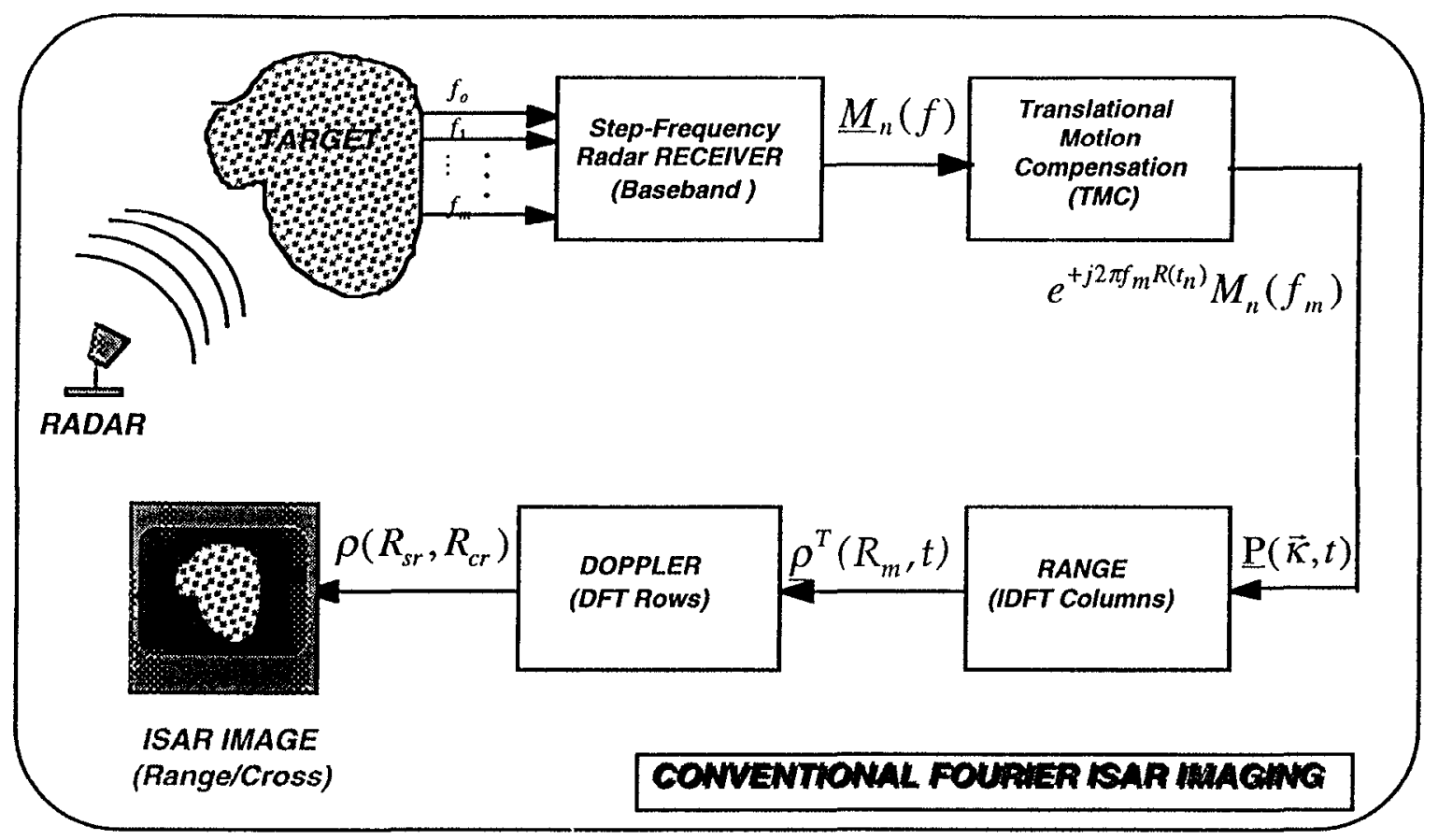

Figure 2. ISAR Processing for a Constant Doppler (Slow Moving) Target.

and that variations in Doppler lead to variations in cross-range. If we assume that the Doppler space is subdivided into small range-Doppler cells, $\left(\Delta R_{s r}, \Delta f_{D}\right)$, then the movement of a particular scatterer within the target must be confined to a single cell during the processing interval $T$, that is, when the rotational velocity is constant, $\omega(t) \rightarrow \omega \times T$. The scatterer will rapidly move through a cell when $\theta= \pm \frac{\pi}{2}$, then $\Delta R_{c r} \geq D \omega T$ or equivalently $\Delta R_{c r} \geq D \theta$, where $D$ is the diameter of a circular bounding region enclosing the entire target and complete set of scatterers describing the corresponding reflectivity function. Thus, from Eq. 31 above we obtain

$$
\Delta R_{c r} \geq \sqrt{\frac{1}{2} D \lambda}
$$

which implies that if this condition is violated due to the target rotation, then the image will be blurred. So, we see that conventional Fourier processing (DFT of range profiles) leads directly to the range/Doppler or equivalent range/cross-range radar image, when the target Doppler is constant. 


\section{Short-Time Fourier Transform Time-Frequency Estimation}

Suppose this is not the case and the Doppler frequency is time-varying, then the condition of Eq. 32 will be violated and radar image reconstruction using the Fourier transform will not be successful and we must resort to other means of so-called rotational motion correction (RMC). There are conventional approaches to this problem, if the motion is not too severe, such as polar reformatting, angle tracking etc. [Weh94,Sch91]; however, in this work we will take the approach of Chen [Che97] and investigate timefrequency estimation as an alternative.

To see this recall that prior to performing the DFT or transforming to range-Doppler space, we have the range profiles of $\rho\left(R_{s r}, t_{n}\right) ; n=0, \cdots, N-1$. Now suppose we temporally window this reflectivity function and calculate its discrete Fourier transform over the finite window (of length $L$ ), then we obtain the so-called short time Fourier transform (STFT) of the profile for each time instant (see Figure 3 below), that is,

$$
\mathrm{P}\left(R_{m}, f_{D}(k), t_{n}\right)=\sum_{\ell=o}^{L-1} \rho\left(R_{m}, t_{n+\ell}\right) w\left(t_{\ell}\right) e^{-j 2 \pi f_{D}(k) t_{\ell}}, \quad k=0, \cdots, K-1
$$

where $f_{D}(k)=\left(\frac{2 \pi k}{L}\right)$, is the discrete Doppler frequency. Thus, if we select a window of length $L$ and slide it through the $m$-th range cell (time) series over each time index, $t_{n}, n=0, \cdots, N-1$; then we can create a set of cell spectrograms, $\quad\left\{P_{m}\left(f_{D}, t\right)\right\}, m=0, \cdots, M-1$; each of which is an $M x K$ range/Doppler image of the scatterers. That is, at each time instant (or window), we "stack" or order all of the corresponding spectra ( $n$-th column of spectrogram') by range cell as

$$
\rho_{n}\left(R_{s r}, f_{D}\right) \equiv\left[\begin{array}{c}
\underline{P}_{o}^{T}\left(f_{D}, t_{n}\right) \\
\vdots \\
\underline{P}_{M-1}^{T}\left(f_{D}, t_{n}\right)
\end{array}\right] ; \quad n=0, \cdots, N-1
$$

therefore, at each $n$, we create the range/Doppler reflectivity image, $\rho_{n}\left(R_{s r}, f_{D}\right)$ composed of the spectra, $\underline{\mathrm{P}}_{m}^{T} \in \mathbf{C}^{\mathbf{l} \times N}$. Unfortunately, the STFT temporal resolution is not $t_{n}$, but at the resolution of the window length $L$ because Doppler processing requires the scatterer to remain in the range bin over this window. This constraint has led to the application of time-frequency 
estimators to the radar imaging problem. We will apply the STFT to our synthesized data set as well as a parametric estimator developed next.

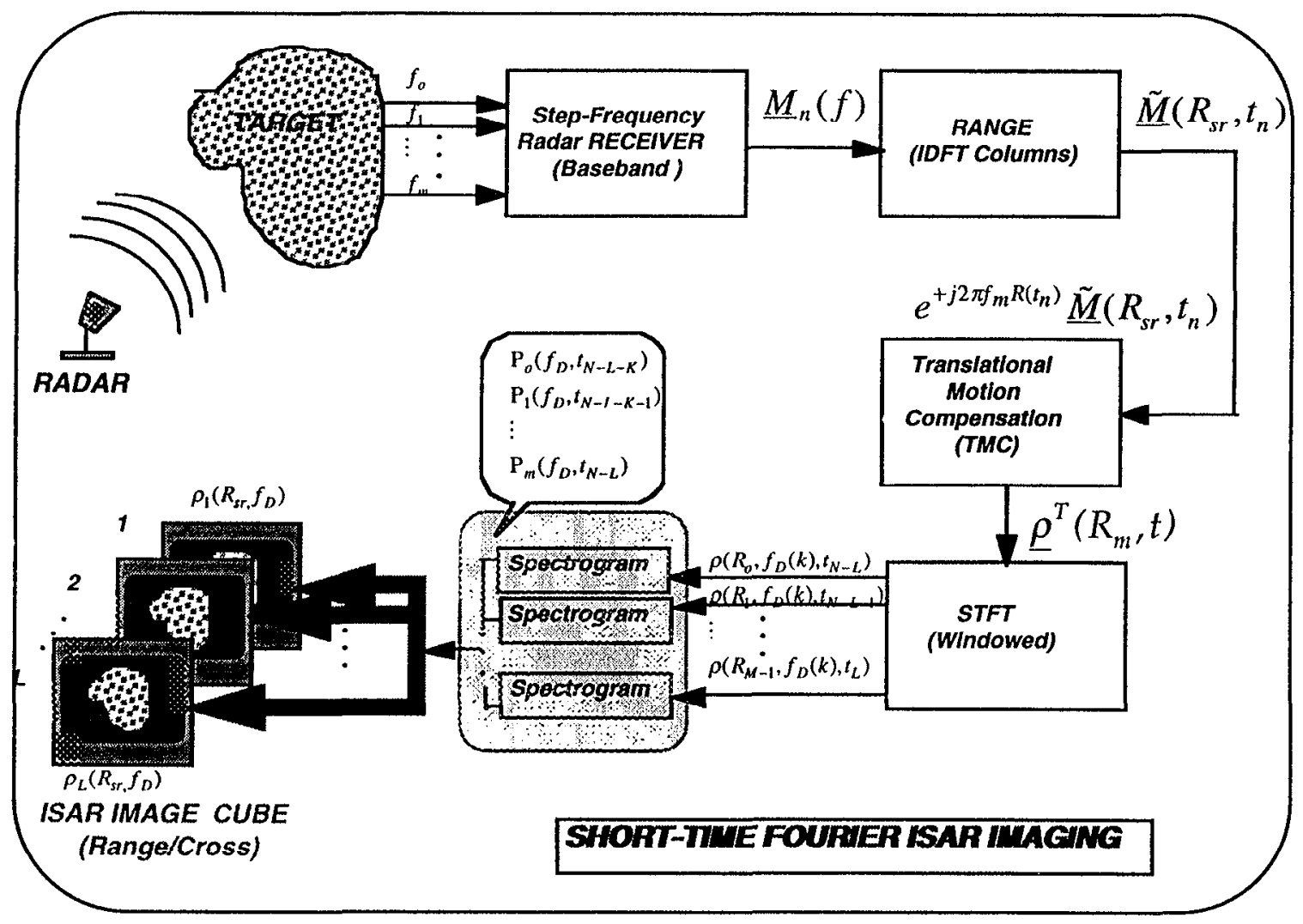

Figure 3. ISAR IMAGE CUBE: Windowed Spectrogram Estimator using STFT (or MVDR/MEM estimators).

\section{Parametric Time-Frequency Estimation}

Suppose we parametrically model the target reflectivity by an instantaneous time-frequency representation specified by an autoregressive moving average (ARMA) model. This model takes the general difference equation form, $\operatorname{ARMA}\left(N_{a}, N_{c}\right)$ given by

$$
A_{m}\left(q^{-1}, t_{n}\right) \rho_{m}\left(t_{n}\right)=C_{m}\left(q^{-1}, t_{n}\right) \varepsilon_{m}\left(t_{n}\right)
$$


for the range (cell) time series, $\rho_{m}\left(t_{n}\right) \equiv \rho\left(R_{m}, t_{n}\right)$, contaminated with zeromean, white gaussian noise, $\varepsilon_{m} \sim N\left(0, \sigma_{m}^{2}\right)$ and the corresponding $m$-th range cell polynomials at the $t_{n}$ instant defined by

$$
\begin{aligned}
& A_{m}\left(q^{-1}, t_{n}\right) \equiv 1+a_{m 1}\left(t_{n}\right) q^{-1}+\cdots+a_{m N_{a}}\left(t_{n}\right) q^{-N_{a}-1} \\
& C_{m}\left(q^{-1}, t_{n}\right) \equiv c_{m o}+c_{m 1}\left(t_{n}\right) q^{-1}+\cdots+c_{m N_{a}}\left(t_{n}\right) q^{-N_{c}-1} .
\end{aligned}
$$

Here the backward shift or delay operator is defined by, $q^{-i} \rho_{m}\left(t_{n}\right) \equiv \rho_{m}\left(t_{n-i}\right)$, and therefore, we can write Eq. 35 simply as

$$
\rho_{m}\left(t_{n}\right)=-\sum_{k=1}^{N_{a}-1} a_{m k}\left(t_{n}\right) \rho_{m}\left(t_{n-k}\right)+\sum_{k=0}^{N_{c}-1} c_{m k}\left(t_{n}\right) \varepsilon_{m}\left(t_{n-k}\right) .
$$

If we take the DFT of the difference equation, then we obtain the instantaneous transfer function (ignoring stochastic aspect)

$$
H_{m}\left(e^{j 2 \pi f D}, t_{n}\right)=\frac{\mathrm{P}_{m}\left(e^{j 2 \pi f D}, t_{n}\right)}{\mathrm{E}_{m}\left(e^{j 2 \pi f D}, t_{n}\right)}=\frac{C_{m}\left(e^{j 2 \pi f D}, t_{n}\right)}{A_{m}\left(e^{j 2 \pi f D}, t_{n}\right)}
$$

or more appropriately the corresponding instantaneous power spectrum given by

$$
S_{m}\left(f_{D}, t_{n}\right) \equiv\left|H_{m}\left(e^{j 2 \pi f D}, t_{n}\right)\right|^{2}=\left|\frac{C_{m}\left(e^{j 2 \pi f D}, t_{n}\right)}{A_{m}\left(e^{j 2 \pi f D}, t_{n}\right)}\right|^{2}
$$

If we "stack" the spectra according to range cell at each time instant, then we obtain the required range/Doppler image $(M x K x N)$-cube discussed previously as

$$
\mathrm{P}\left(R_{s r}, f_{D}, t_{n}\right) \equiv\left[\begin{array}{c}
S_{o}\left(f_{D}, t_{n}\right) \\
\vdots \\
S_{M-1}\left(f_{D}, t_{n}\right)
\end{array}\right], \quad n=0, \cdots, N-1,
$$

with each cube element a 2D-range/Doppler or range/cross-range image at the specified time instant. 
So we see that if we use the parametric $\operatorname{ARMA}\left(N_{a}, N_{c}\right)$ representation of the range profiles and transform them to the spectral domain, then we can obtain an instantaneous representation of the range/Doppler or range/crossrange image with the added advantage of the increased resolution inherited by the parametric representations along with the instantaneous temporal estimates. We summarize the instantaneous parametric processing operations applied to the SFWF data in Figure 4.

There are a wealth of ARMA algorithms available in the literature [Can86,Lju87,Sod89], but since we are primarily interested in estimating the spectrum at each time instant, we confine our choices to those that are recursive-in-time enabling us to achieve our goal without the loss of temporal resolution evolving from window-based methods such as the STFT. Recursive-in-time algorithms (real) all take on the following generic form for the $m$-th range profile:

$$
\begin{aligned}
& \underline{\hat{\alpha}}_{m}\left(t_{n+1}\right)=\underline{\hat{\hat{\alpha}}}_{m}\left(t_{n}\right)+\underline{G}_{m}\left(t_{n}\right) e_{m}\left(t_{n}\right) \\
& \left.e_{m}\left(t_{n}\right)=\rho_{m}\left(t_{n}\right)-\hat{\rho}_{m}\left(t_{n}\right)=\rho_{m}\left(t_{n}\right)-\underline{\varphi}_{m}^{T}\left(t_{n}\right) \underline{\hat{\alpha}}_{m}\left(t_{n}\right) \quad \text { [prediction error }\right]
\end{aligned}
$$

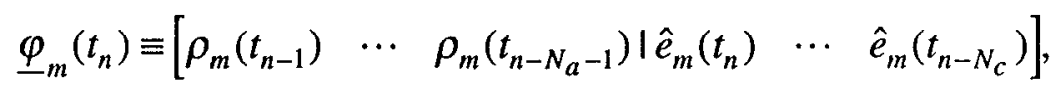

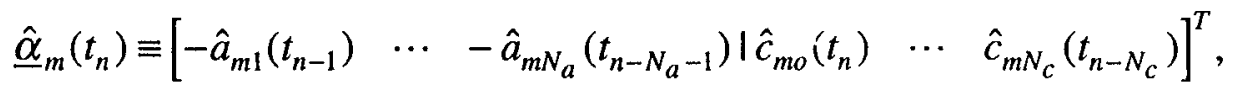

with $\underline{G}_{m}$ the so-called gain or weighting vector and the $\wedge$ symbol defining the "best" (minimum error variance) estimate at the specified time. There are also many variations and forms of this basic recursion [Can86], but here we will limit our application to the recursive prediction error method (RPEM) based on a local Gauss-Newton optimization method (see [Lju87,Sod89] for details). We will evaluate the performance of this approach and compare it to the STFT in the next section. 


\section{ISAR SIMULATION}

In this section we discuss the simulator developed to generate ISAR signals used in the subsequent processing examples. We are developing a simulation based on the target reflectivity function of a target vehicle. The basic model consists of decomposing the 2D-reflectivity function into a set of ideal discrete point scatterers, $\rho(x, y) \rightarrow \rho\left(x_{i}, y_{j}\right)$, that is,

$$
\rho\left(x_{i}, y_{j}\right) \equiv \sum_{i} \sum_{j} \beta_{i j} \delta\left(x-x_{i}, y-y_{j}\right) \Delta x \Delta y,
$$

where $\beta_{i j}$ is the reflectivity of the $i j-$ th point scatterer. Next returning to the geometry of Figure 1 and realizing that the translational and rotational motion of the target can be modeled relative to its center-of-rotation, we develop the basic model. A model of the ISAR system and its corresponding image reconstruction is created.

The ISAR system model is developed as a simulation which supposes a frequency stepped radar at the origin of a coordinate system and a number of point scatterers at various locations within the system as shown in Figure 1. The point scatterers rotate about the target central point, (COR) as a function of time. The COR is also allowed a radial degree of freedom so that the point scatterers travel towards or away from the radar as a function of time with constant velocity and acceleration parameters. The full motion of an object is then traveling with some speed relative to the radar and rotating within the ISAR beam.

The SFWF radar simulator operates by first assigning locations or grid coordinates to the COR and point scatterers. Each point scatterer is defined in polar coordinates by a vector relative to the COR, the $i$-th scatterer location is

$$
d_{i}\left(t_{n}\right)=\sqrt{x_{i}^{2}\left(t_{n}\right)+y_{i}^{2}\left(t_{n}\right)}
$$

along with its corresponding scatterer angle as

$$
\theta_{i}(t)=\arccos \frac{x_{i}\left(t_{n}\right)}{d_{i}\left(t_{n}\right)} .
$$




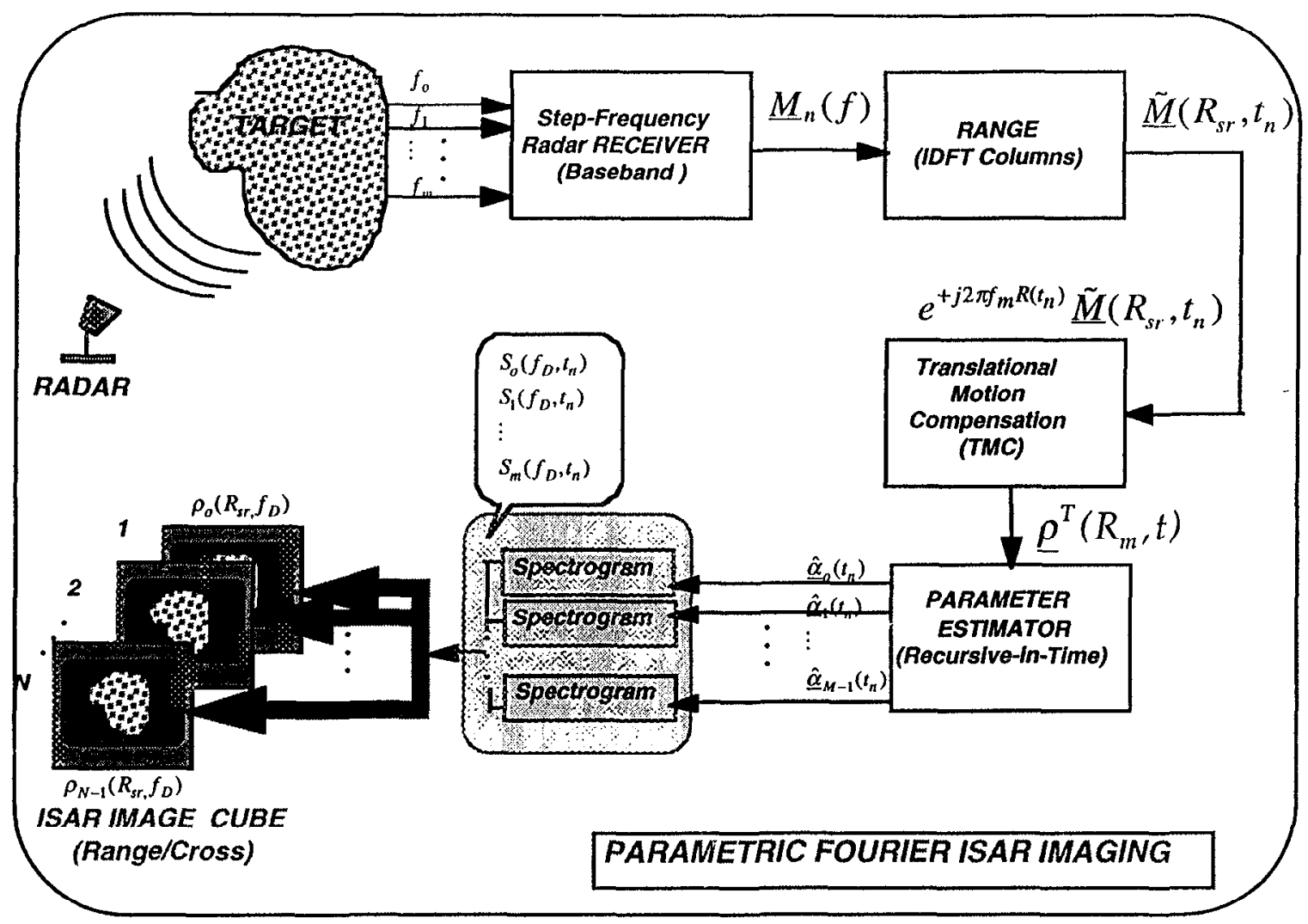

Figure 4. ISAR IMAGE CUBE: Instantaneous Spectrogram Estimator using Recursive-in-Time, Parameter Estimator.

The translational motion of the target COR in $2 \mathrm{D}$ is defined by the timevarying coordinates $\left(X\left(t_{n}\right), Y\left(t_{n}\right)\right)$ as

$$
\begin{aligned}
& X\left(t_{n}\right)=X_{o}+v_{x} t_{n}+a_{x} \frac{t_{n}^{2}}{2}, \\
& Y\left(t_{n}\right)=Y_{o}+v_{y} t_{n}+a_{y} \frac{t_{n}^{2}}{2},
\end{aligned}
$$

and therefore

$$
R_{\mathrm{COR}}\left(t_{n}\right)=\sqrt{\mathrm{X}^{2}\left(t_{n}\right)+Y^{2}\left(t_{n}\right)} .
$$

Similarly, the target COR is given as before by 


$$
\Theta_{C O R}\left(t_{n}\right)=\Theta_{o}+\omega t_{n}+\alpha \frac{l_{n}^{2}}{2}
$$

which leads to the individual scatterer rotations (in $\mathrm{XY}$-coordinates) relative to the COR as

$$
\Theta_{i}\left(t_{n}\right)=\Theta_{C O R}\left(t_{n}\right) \pm \theta_{i}(t)
$$

The ISAR scatterer simulation is then accomplished by first calculating the COR radial and rotational trajectories using Eqs. 46 and 47 , and then adjusting each individual scatterer in target space using the relations

$$
\begin{aligned}
& X_{i}\left(t_{n}\right)=X\left(t_{n}\right)+\left(d_{i}\left(t_{n}\right) \sin \Theta_{i}(t)-d_{i}\left(t_{n}\right) \cos \Theta_{i}(t)\right), \\
& Y_{i}\left(t_{n}\right)=Y\left(t_{n}\right)+\left(d_{i}\left(t_{n}\right) \cos \Theta_{i}(t)-d_{i}\left(t_{n}\right) \sin \Theta_{i}(t)\right) .
\end{aligned}
$$

The range trajectory of each scatterer (relative to the radar observer) is then simply

$$
r_{i}\left(t_{n}\right)=\sqrt{X_{i}^{2}\left(t_{n}\right)+Y_{i}^{2}\left(t_{n}\right)} .
$$

Using these relations, the complex received signal from the synthesized SFWF radar is therefore, given by the superposition of all the scatterers composing the target reflectivity function

$$
p_{m}\left(t_{n}\right)=\sum_{i=1}^{N_{s}} e^{-j 2 \pi f_{m} r_{i}\left(t_{n}\right)} ; \quad m=0, \cdots, M-1, n=0, \cdots, N-1 ;
$$

This simulator is able to synthesize the returns from multiple point scatterers located at given positions relative to the COR. The scatterers can also be pre-programmed to travel with rotation and radial velocity to simulate the actions of a moving target within the ISAR beam. It synthesizes a SFWF radar illumination discussed in Section II. The simulator takes as parameters the radial velocity and acceleration as well as rotational velocity and acceleration and computes the course and position of each individual scatterer along the path. From these positions, the range is calculated for each point scatterer (Eq. 50) as well as its phase (Eq. 51) . 
Parameters are assigned to give functions for the radial and rotational motions about the COR for each time sample during the simulation. With these parameters, the exact grid coordinate $\left(X_{i}(t), Y_{i}(t)\right)$ of each point scatterer can be calculated (see Eq. 49). From these results, the range to target is easily computed for each scatterer at each time sample (Eq. 50). Once the full motion of the target is simulated, the stepped frequency radar signal is created and applied to the moving target. A set of frequencies are defined as in Eq. 17 along with the frequency step, $\Delta f$. This step must be chosen carefully, so that frequency aliasing does not become problematic. The phase for each point target can now be calculated by applying the stepped frequency waveform to the targets at each time sample. Using the analytic signal, the return from a radar stepped frequency pulse is defined in Eq. 51. After computing for all $m$ and $t_{n}$, the time varying or instantaneous frequency response is the result. The data are collected and saved for future processing.

The following simulation parameters were used to synthesize the translational and rotational motion of a vehicle observed by the radar:

Table 1. Vehicle Simulation Problem: SFWF Radar Simulation Parameters.

\begin{tabular}{|l|c|}
\hline \multicolumn{1}{|c|}{ PARAMETER } \\
\hline Propagation Velocity $(c)$ & $3 \times 10^{8}(\mathrm{~m} / \mathrm{s})$ \\
\hline Pulse Repetition Frequency (PRF) & $1 \times 10^{4}(\mathrm{~Hz})$ \\
\hline Frequency Step-Size $(\Delta f)$ & $1 \times 10^{7}(\mathrm{~Hz})$ \\
\hline Center Frequency $\left(f_{c}\right)$ & $50 \times 10^{9}(\mathrm{~Hz})$ \\
\hline No. Frequency Bins $(\mathrm{M})$ & 256 \\
\hline No. of Range Samples & 256 \\
\hline No. of Azimuth Bins (L) & 64 \\
\hline No. of Azimuth Samples & 128 \\
\hline
\end{tabular}

Table 2. Vehicle Simulation Problem: COR/Scatterer Simulation Parameters.

\begin{tabular}{|c|c|c|c|c|c|c|c|c|}
\hline & $R_{o}(m)$ & $v_{R}(m / s$ & $a_{R}\left(m / s^{2}\right.$ & $\theta_{o}(r a d)$ & $\omega(r / s)$ & $\alpha\left(r / s^{2}\right)$ & $x(m)$ & $y(m)$ \\
\hline COR & 714 & 0.015 & 0.0005 & 0.0 & 0.07 & 0.002 & 508.1 & 505.1 \\
\hline Scat 1 & & & & & & & 0.5 & 0.5 \\
\hline Scat 2 & & & & & & & -0.5 & -0.5 \\
\hline Scat 3 & & & & & & & -0.5 & 0.5 \\
\hline Scat 4 & & & & & & & 0.7 & -0.7 \\
\hline
\end{tabular}


The results of the low-noise simulation are shown in Figure 5 below. Here the vehicle is represented by four point scatterers and their corresponding trajectories in the range-time plane are obtained by IDFT processing of the SFWF radar returns. Note the rotational sinusoidial scatterer traces on the translational parabolic arcs. Each scatterer travels at a different angular response relative to the overall target COR.

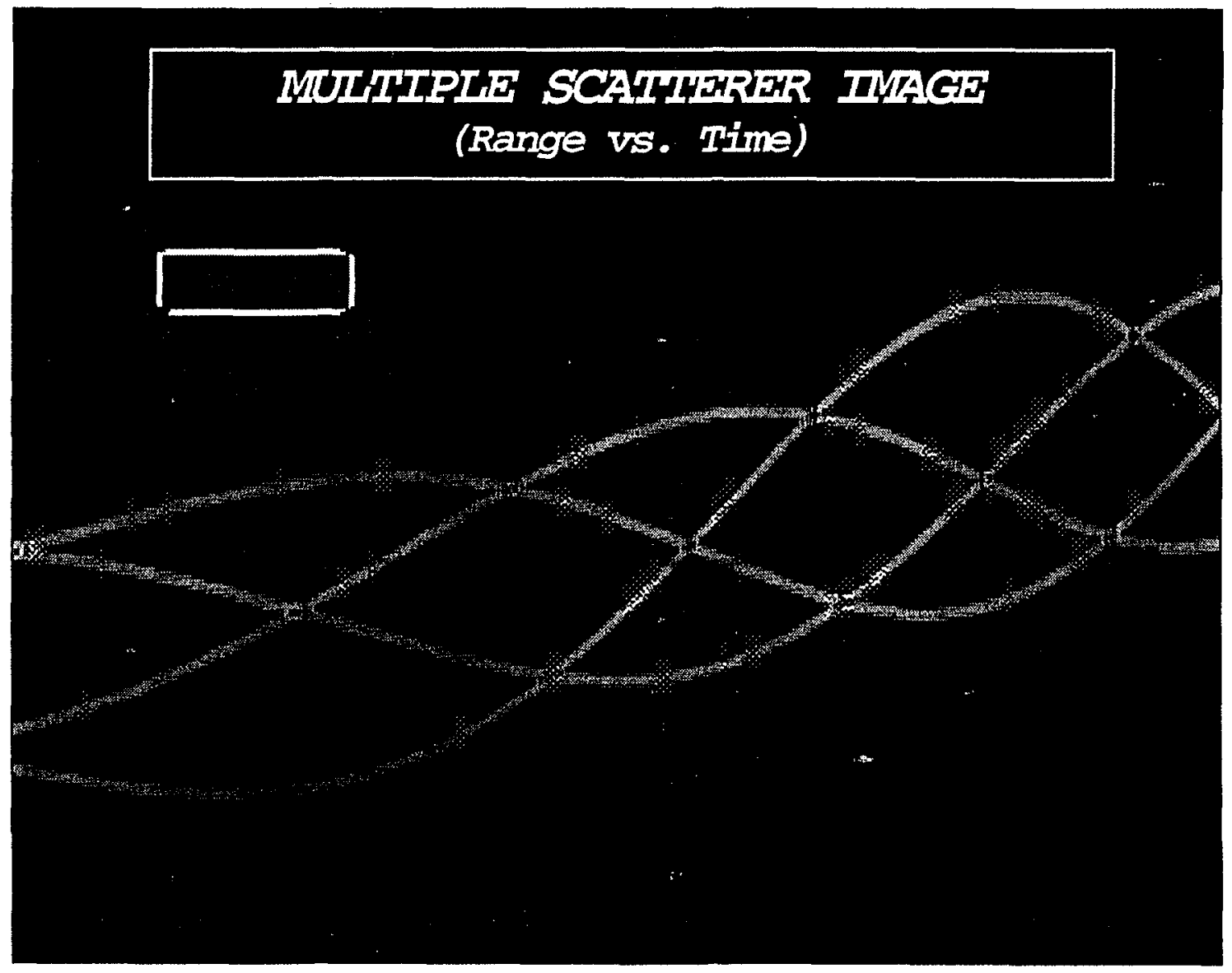

Figure 5. Vehicle Simulation as Four (4) Point Scatterers with both Translational and Rotational Motion: Range $(6.375 m) \times$ Time( $37.5 \mathrm{sec})$. 


\section{RANGE-ENVELOPE TMC ESTIMATION}

To obtain cross-range resolution, each time-slice within the window must be aligned so that the range is equivalent. TMC is accomplished by estimating the COR from the range profile data. First, the upper and lower range limits are estimated using threshold filtering for each profile (column) thereby defining its so-called range-envelope. The middle or average pixel between the upper and lower bound points is estimated to be the COR for the given profile (column) and therefore becomes the amount of rotation necessary to align the range bins in consecutive time samples. A second order polynomial model is fit to the estimated COR and compared with the original parameters used in the simulator. The effects of noise can be seen by directly comparing the original parameters with the estimated parameters after random noise has been added to the system. Each time sample is rotated accordingly to obtain the motion-compensated image window.

We developed a range-envelope TMC algorithm to estimate the translational motion of the COR from each (uncompensated) range profile, $\underline{\tilde{M}}\left(R_{s r}, t_{n}\right) ; n=0, \cdots, N-1$ of Eq. 26 . That is, after thresholding the uncompensated range profiles at level $\tau$, the mean value of each profile (column) is estimated based on the principle that each scatterer follows the same transversal motion of the COR,

$$
\tilde{R}\left(t_{n}\right)=E\left\{\underline{\tilde{M}}_{\tau}\left(R_{s r}, t_{n}\right)\right\} ; \quad n=0, \cdots, N-1,
$$

yielding a noisy 1D-measurement sequence of the average range-envelope, $\left\{\tilde{R}\left(t_{n}\right)\right\}$. Next a minimum variance estimator is designed to estimate the transversal position, velocity and acceleration coefficients using a second order polynomial model of Eq. 5 . We use the range polynomial model

$$
R(t)=R_{o}+v_{R} t+a_{R} \frac{t^{2}}{2}=\left[\begin{array}{lll}
1 & t & \frac{t^{2}}{2}
\end{array}\right]\left[\begin{array}{c}
R_{o} \\
v_{R} \\
a_{R}
\end{array}\right]=\underline{\tau}^{T}(t) \underline{\varphi},
$$

which can be modeled stochastically with additive gaussian noise, $n \sim N\left(0, \sigma_{n}^{2}\right)$, as

$$
\tilde{R}(t)=\underline{\tau}^{T}(t) \underline{\varphi}+n(t)
$$

The translational estimator follows by letting $t \rightarrow t_{n} ; n=0, \cdots, N-1$, and "stacking" the equations of Eq. 54 to create the batch measurement model, 


$$
\underline{\tilde{r}}=\mathrm{T} \underline{\varphi}+\underline{n},
$$

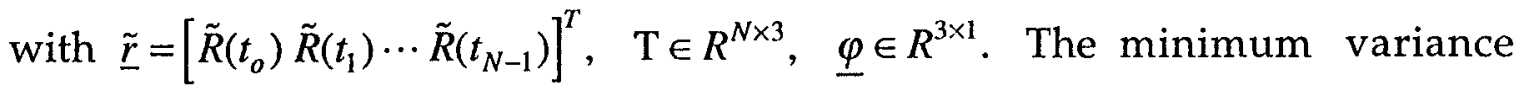
solution to this problem is well-known [Can86] and can be solved robustly using the singular value decomposition (SVD) algorithm to yield

$$
\hat{\underline{\varphi}}_{m \nu}=\left(\mathrm{T}^{T} \Sigma^{-1} \mathrm{~T}\right)^{-1} \mathrm{~T}^{T} \Sigma^{-1} \underline{\underline{\underline{r}}}
$$

where $\Sigma \equiv \operatorname{cov}(\underline{n})$. The minimum variance estimate of the corresponding translational trajectory for TMC is given by

$$
\hat{R}_{m v}\left(t_{n}\right)=\underline{\tau}^{T}\left(t_{n}\right) \underline{\hat{\varphi}}_{m v}=\hat{R}_{o}+\hat{v}_{R} t_{n}+\hat{a}_{R} \frac{t_{n}^{2}}{2} .
$$

We summarize the Range-Envelope TMC algorithm for ISAR SFWF data as:

\section{RANGE-ENVELOPE TMC ALGORITHM}

Step 1. Perform the columnwise 1D-IDFT of the measured SFWF data, $\underline{M}_{n}(f)$ to obtain the uncompensated range profiles, $\underline{\tilde{M}}\left(R_{s r}, t_{n}\right)$ of Eq. 26 ;

Step 2. Threshold filter the range profile data to bound the envelope limits obtaining, $\underline{\underline{M}}_{\tau}\left(R_{s r}, t_{n}\right)$;

Step 3. Estimate the average value of the thresholded profiles to produce the 1D measurement sequence, $\tilde{R}\left(t_{n}\right)$;

Step 4. Estimate the sample noise variance, $\hat{\sigma}_{m}^{2}$ from any "noise only" row of the raw range profile image, and create the noise covariance matrix, $\Sigma=\operatorname{diag}\left(\sigma_{m}^{2}\right)$;

Step 5. Perform the minimum variance estimation to obtain, $\underline{\hat{\varphi}}_{M V}$ of Eq. 56;

Step 6. Perform the TMC at each time instant using $\hat{R}_{M V}\left(t_{n}\right)$ in Eq. 57. 
We applied this processor to the simulated data set of Figure 5 with additive noise, $\Sigma=\operatorname{diag}\left(\sigma_{n}^{2}\right), \sigma_{n}^{2}=1 \times 10^{-6}$. The following estimates were obtained:

Table 3. Minimum Variance Estimates of Translational Trajectory Parameters for TMC.

\begin{tabular}{|c|c|c|}
\hline PARAMETER & True & Estimate \\
\hline & & \\
\hline Position & 714 & 708 \\
\hline Velocity & 0.015 & 0.0105 \\
\hline Acceleration & 0.0005 & 0.00035 \\
\hline
\end{tabular}

These estimates are then used to perform the TMC of the range data using Eq. 57 at each time step. Note that a recursive rather than batch parameter estimator can also be used to estimate the instantaneous radial velocity of the target for TMC (see Eq. 56). Any error in the estimation of the radial velocity results in a shift in subsequent images.

We show the results of the TMC algorithm on the synthesized data in Figure 6. In $6 \mathrm{a}$, we see the estimate of the range polynomial model (solid line) superimposed on the extracted envelope data (asterisks) of Eq. 52. The fit is quite good as expected in this high SNR case which demonstrates the concepts. In $6 \mathrm{~b}$ we observe the results of applying the dynamic TMC to the range profile data of Figure 5 demonstrating the removal of the time-varying range trajectory. Next we process this corrected data with the simple STFT time-frequency estimator. 


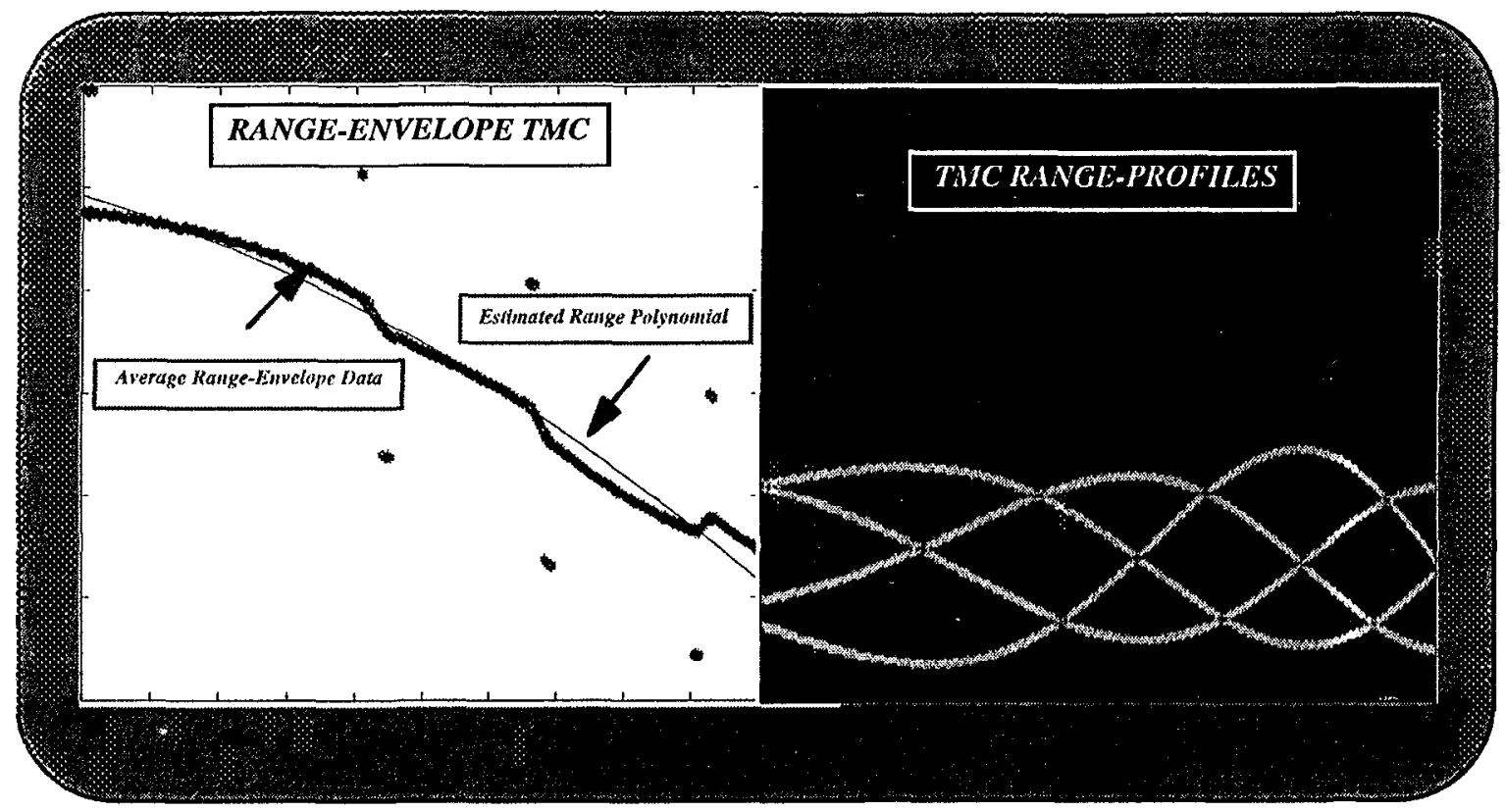

Figure 6. Translation Motion Compensation for Vehicle Simulation: (a) Range-Envelope TMC results. (b) Dynamically Compensated Range Profiles.

\section{STFT ISAR Imaging}

If the data are arranged in the temporal reflectivity matrix of Eq. 28 such that the frequency increments from a particular time interval form the columns of a matrix, and each column is placed from left to right with increasing time, a time-varying image can be formed. That is, an image with time-dependent frequency response on the vertical axis and time increments on the horizontal axis. Taking the Fourier transform of each column yields the spatial locations of point targets at that time interval. Processing each column in this way yields the time-varying spatial response in the range direction of Figure $6 b$.

STFT Doppler processing (see Figure 3 for details) is then used to obtain azimuthal, or cross-range resolution. Since the Doppler frequencies for each point target are a function of time, a time window must be selected such that Doppler frequency smearing is minimized. At this point, the Doppler signature can be computed by calculating the spectrum of the range bin and shifting so that the DC component appears in the center of the image. Various methods of determining the spectrum can be used, each with it's own effects. The FFT-based STFT can be used but is less effective if noise is present in the system. 
The results of applying the STFT processor to the TMC data is shown in the montage of Figure 7 where each window is shown at approximately 1.5 $\mathrm{sec}$ frame rate of the $37.5 \mathrm{sec}$ simulation. The important point to note from the figure is that the vehicle target represented by the four (4) point scatterers can be observed in each frame with little smearing as would have been the case if the conventional Fourier processor of Figure 2 was applied. In fact, the rotational motion can clearly be observed in the montage demonstrating the effectiveness of the time-frequency approach. Unfortunately as mentioned before, the STFT is much less effective in a noisy environment and therefore more sophisticated processors must be employed in real world situations. In this work, we limit our attention to the parametric approach discussed next and applied to the simulated target data.

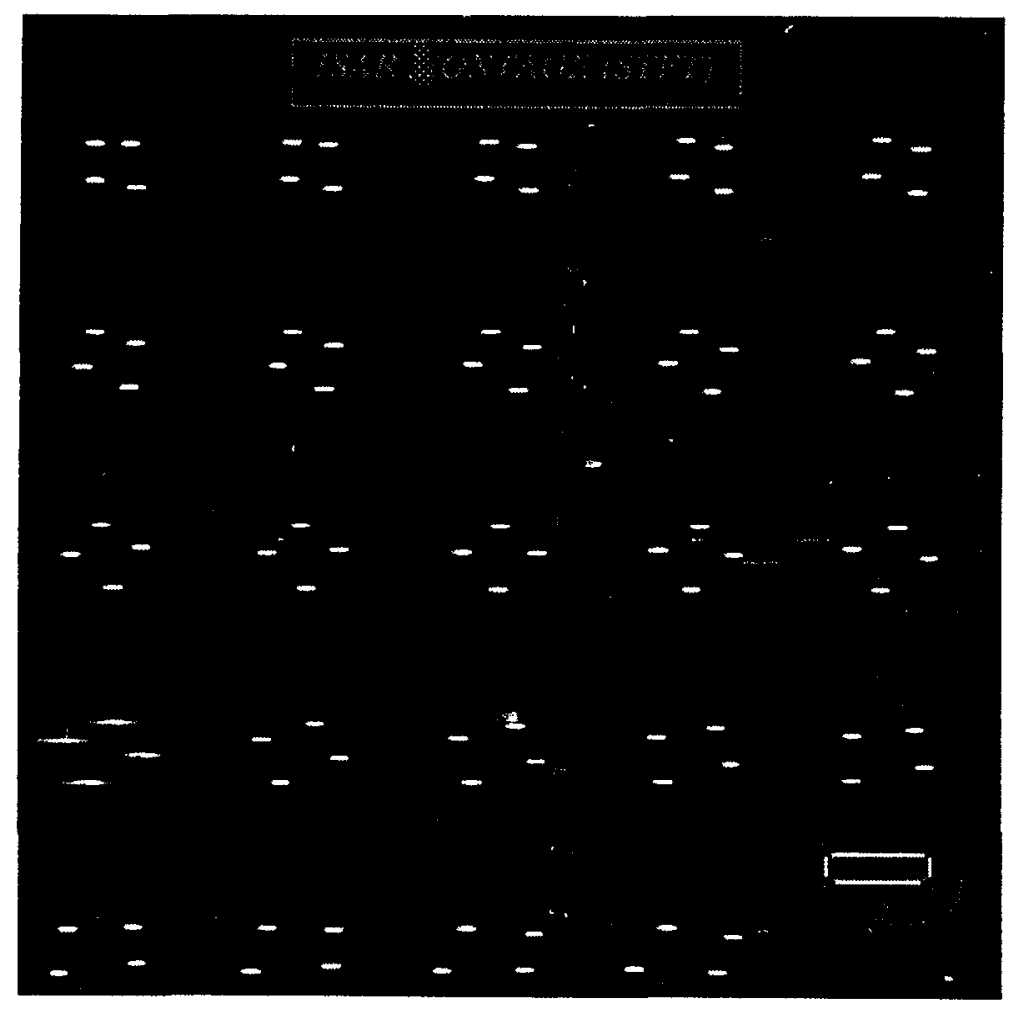

Figure 7. ISAR Vehicle Image Reconstruction: STFT processing montage showing target motion at a $1.5 \mathrm{sec}$ frame rate (start at upper left corner).

\section{PARAMETRIC (RECURSIVE-IN-TIME) ISAR IMAGING}

Our approach is to develop the recursive (in-time) spectral estimator discussed in the previous section which does not depend on time-windows. 
A recursive spectral estimator can also be used to compute the Doppler spectra. First, the Doppler spectra can be computed and stored as a set of coefficients rather than a full vector. This can be an advantage for real time systems which rely on compressed data outputs. Secondly, the spectral coefficients represent a model of the instantaneous spectrum and can be expanded above the maximum resolution dictated by the Doppler bandwidth.

\section{PHASE I' WORK}

\section{SUMMARY}

In this report we have developed a recursive-in-time approach to estimating the instantaneous Doppler spectra for creating ISAR range/Doppler or range/cross-range images. We developed the required background theory to show precisely where the ISAR evolves from a steppedfrequency radar and how the final image can be improved using timefrequency estimation techniques following the work of Chen [Che97]. It was shown that for any significant target motion the corresponding Doppler frequency is directly effected. If this motion is not accounted for, then the Doppler information is smeared causing the final target image to be blurred. After basebanding the received SFWF measurements, target motion compensation (TMC) was performed to align the range as in SAR. The TMC estimator (minimum error variance) developed employs a polynomial parameter estimator to "fit" the dynamic translational components of the target's center of rotation (COR) to remove the radial velocity and acceleration effects. After applying the inverse Fourier transform to extract the various range profiles, the time-frequency estimation techniques were applied to extract the target images. Here the STFT was used in the noise-free environment to demonstrate the basic principle, then as the signal-to-noise ratio (SNR) was decreased, more sophisticated parametric processors will be developed to extract the desired target reflectivity image.

Finally we will develop the basic recursive-in-time approach (see Section III) and selected the recursive prediction error method (RPEM) of Ljung to apply to the ISAR problem (see [Lju87]). It will be shown that not only could it reproduce the STFT results, but also function quite well in low SNR environments.

\section{ACKNOWLEDGEMENTS}

This work was supported by the Office of the Chief of Naval Research under contract N00014-97-F-0397. The Project Officer was Dr. W. Micelli who motivated the basic vehicular ISAR problem and supported this effort. We would also like to acknowledge the various technical discussions with Dr. V. 
Chen of the Naval Reseach Laboratory as well as access to simulated and measured data sets he provided.

\section{REFERENCES}

[Boa92] B. Boashash, Editor. Tim-Frequency Signal Analysis: Methods and Applications. New York: Wiley, 1992.

[Bo190] P. L. Bolger. Radar Principles with Applications to Tracking Systems. New York: Wiley, 1990.

[Can86] J. V. Candy. Signal Processing: The Model-Based Approach. New York: McGraw-Hill, 1986.

[Che80] C. C. Chen. "Target-motion-induced radar imaging." IEEE Trans. Aero. E Eletronic. Sys., AES-16, (1), 2-14, 1980

[Che95] V. C. Chen. "Radar ambiguity function, time-varying matched filter and optimum wavelet correlator." Opt. Eng., 33 (7), 22122217, 1994.

[Che97] V. C. Chen. "Applications of time-frequency processing to radar imaging." Opt. Eng., 36 (4), 1152-1161, 1997.

[Coh95] L. Cohen. Time-Frequency Analysis. Englewood Cliffs, NJ: Prentice-Hall, 1995.

[Cur91] J. C. Curlander and R. N. McDonough. Synthetic Aperture Radar: Systems and Signal Processing. Englewood Cliffs, NJ: Prentice-Hall, 1991.

[Joh96] B. Johnston. Time-Frequency Analysis of Synthetic Aperture Radar Signals. LLNL Report: UCRL-LR-125080, 1996.

[Lju87] L. Ljung. System Identification: Theory for the User. Englewood Cliffs, NJ: Prentice-Hall, 1987.

[Men91] D. L. Mensa. High Resolution Radar Cross-Section Imaging. Boston, MA: Artech House, 1991.

[Qia96] S. Qian and D. Chen. Joint Time-Frequency Analysis: Methods and Applications. NJ: Prentice-Hall, 1996.

[Rih96a] A. W. Rihaczek and S. J. Hershkowitz. Principles of High Resolution Radar. Boston, MA: Artech House, 1996.

[Rih96b] A. W. Rihaczek and S. J. Hershkowitz. Radar Resolution and Complex-Image Analysis. Boston, MA: Artech House, 1996.

[She93] J. A. Scheer and J. L. Kurtz, Eds. Coherent Radar Performance Estimation. Boston, MA: Artech House, 1993.

[Sod89] T. Soderstrom and P. Stoica. System Identification. New York: Prentice-Hall International, 1989.

[Sou94] M. Souekh. Fourier Array Imaging. Englewood Cliffs, NJ: Prentice-Hall, 1994.

[Ste91] B. D. Steinberg and H. M. Subbaram. Microwave Imaging Techniques. New York: Wiley, 1991. 
[Wa180] J. L. Walker. "Range-Doppler imaging of rotating objects." IEEE Trans. Aero. \& Eletronic. Sys., AES-16, (1), 23-52, 1980

[Weh94] D. R. Wehner. High Resolution Radar. Boston, MA: Artech House, 1994. 


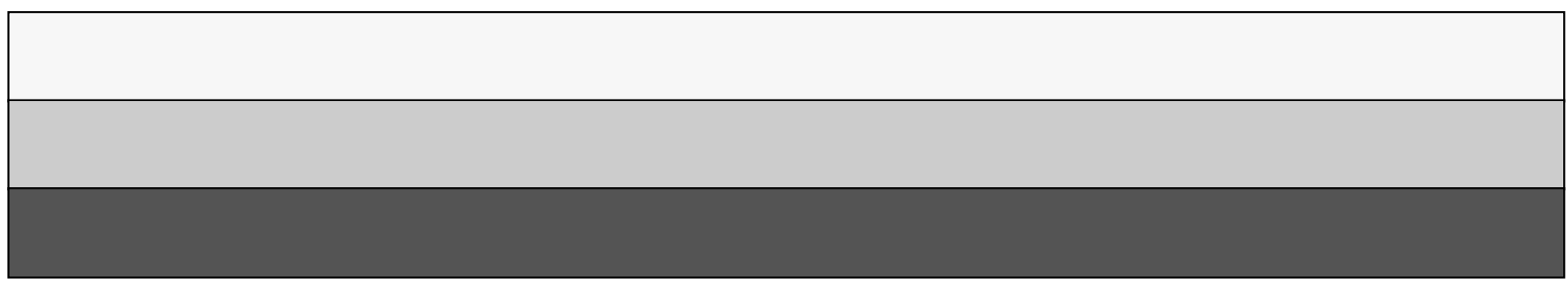

